\title{
Consequências do ronco não-tratado
}

\author{
Consequences of untreated snoring \\ Flávio José Magalhães da Silveira, Ricardo Luiz de Menezes Duarte
}

\begin{abstract}
Resumo
Em alguns estudos, o ronco tem sido associado com um risco aumentado de hipertensão, doença cardíaca isquêmica e acidente vascular encefálico. Os mecanismos são desconhecidos, mas provavelmente mediados pela apneia obstrutiva do sono. Contudo, a maioria dos roncadores não tem apneia do sono. Se o ronco, por si só, aumenta o risco de doença cardiovascular, isso ainda permanece controverso.
\end{abstract}

Descritores: Ronco; Doenças cardiovasculares; Acidente cerebral vascular.

\begin{abstract}
In some studies, snoring has been associated with an increased risk of hypertension, ischemic heart disease and stroke. Although the mechanisms involved in these associations are unknown, they are probably mediated by obstructive sleep apnea. Nevertheless, most snorers do not have sleep apnea. Whether snoring itself increases the risk of cardiovascular disease remains controversial.
\end{abstract}

Keywords: Snoring; Cardiovascular diseases; Stroke.

\section{Introdução}

A maioria dos estudos epidemiológicos demonstra que o ronco está associado a um aumento do risco de hipertensão arterial sistêmica, doença arterial coronariana e acidente vascular encefálico. ${ }^{(1-4)} 0$ mecanismo pelo qual o ronco está associado a essas complicações está principalmente associado à presença de SAHOS, uma vez que o ronco intenso quase sempre está presente em SAHOS.(5) De forma independente, SAHOS pode aumentar o risco de hipertensão arterial sistêmica e, de forma aditiva, pode causar hipoxemia intermitente, a qual promove aterosclerose. ${ }^{(6,7)}$ Entretanto, deve-se salientar que a maioria dos roncadores não possui SAHOS. Se o ronco por si só é capaz de aumentar o risco de hipertensão arterial sistêmica e de doença cardiovascular, isso ainda permanece controverso, com uma tendência a se considerar que essa associação realmente possa coexistir. ${ }^{(5,8)}$

\section{Associação do ronco com doença cardiovascular}

Uma vez que a maioria dos roncadores não apresenta SAHOS, não está claro, como descrito acima, se o ronco isoladamente pode aumentar o risco de doença cardiovascular. Em um estudo transversal com 580 pacientes adultos, o ronco isolado foi associado, de forma estatisticamente significativa, com o aumento da pressão arterial sistêmica, independentemente de outras variáveis, como idade, gênero e IMC. A magnitude da associação, contudo, foi menor do que para outros distúrbios respiratórios do sono. Os autores sugerem que o ronco primário faça parte de um processo contínuo de colapso da via aérea superior e de resistência aumentada dessa via até o aparecimento futuro de SAHOS. ${ }^{(9,10)}$

Em um estudo, foi verificado que o ronco, em mulheres, está associado a um aumento pequeno, porém significativo, do risco de doença cardiovascular, independentemente da idade, da história tabágica, do IMC e de outros riscos conhecidos de doença cardiovascular. ${ }^{(11)}$ Esses dados, em mulheres, já tinham sido previamente verificados em homens, através de um estudo prospectivo de 4.388 homens, no qual 149 pacientes foram recentemente diagnosticados com doença cardíaca isquêmica e 42 pacientes foram diagnosticados com acidente vascular encefálico durante os três anos de acompanhamento do estudo. ${ }^{(3)} \mathrm{Em}$ um estudo transversal com 3.847 homens e 3.664 mulheres, foi encontrada uma associação significativa de ronco habitual com angina pectoris em homens (risco relativo $[R R]=2,01$ ), mas com uma fraca associação em mulheres $(\mathrm{RR}=1,23)$. $^{(2)}$

Outro estudo englobou 1.453 pacientes de ambos os gêneros, com idades entre 20 e 70 anos, 
com o objetivo de verificar se havia associação entre infarto agudo do miocárdio e ronco. Os pacientes foram divididos em dois grupos: 40\% de roncadores e $60 \%$ de não roncadores. Entre os roncadores, houve um RR ajustado para infarto agudo do miocárdio (em relação aos não roncadores) de 3,08 (1C95\%: 1,01-9,46). ${ }^{(12)}$

É possível que, por si só, o ronco possa causar complicações cardiovasculares. 0 ronco é acompanhado por um potente esforço inspiratório contra uma faringe semifechada, com grandes variações na pressão pleural e, por conseguinte, afetando, de forma transiente, tanto a pré-carga quanto à pós-carga cardíaca. ${ }^{(13)} \mathrm{Em}$ uma revisão com 19 estudos com populações de adultos roncadores, apesar de resultados conflitantes e controversos, o autor concluiu que os dados não suportam a premissa de que o ronco primário seja um fator de risco independente para complicações vasculares. ${ }^{(8)}$

\section{Associação do ronco com doenças cerebrovasculares}

0 ronco também tem sido considerado como um fator de risco para infarto cerebral, especialmente acidente vascular encefálico que ocorre durante o sono ou nas primeiras horas após o acordar. Da mesma forma que na doença cardiovascular, diversos fatores de confusão (por ex., hipertensão arterial sistêmica, doença cardiovascular, idade, obesidade, tabagismo e uso de álcool) podem ser considerados na associação entre ronco e acidente vascular encefálico, além do fato de que esses dois achados (ronco e infarto cerebral) também se associam com SAHOS. ${ }^{(3,14,15)}$

Um estudo foi elaborado para verificar a associação entre ronco e infarto cerebral, através de um estudo de caso-controle para fatores de risco para acidente vascular encefálico isquêmico em 177 pacientes do gênero masculino com idades entre 16 e 60 anos. 0 resultado de OR de ronco para acidente vascular encefálico isquêmico foi de 2,13 . Se havia concomitância de SAHOS (com sonolência diurna excessiva) e obesidade, o valor de OR subia para 8,0, indicando que o ronco pode ser um fator de risco para acidente vascular encefálico isquêmico, possivelmente pela alta prevalência de SAHOS entre esses pacientes roncadores. ${ }^{(16)}$ Os mecanismos que poderiam explicar o aumento do risco de acidente vascular encefálico isquêmico em roncadores ainda são desconhecidos. Os possíveis mecanismos poderiam ser a alta prevalência de SAHOS em roncadores, a associação com arritmias cardíacas e a diminuição do índice cardíaco. ${ }^{(16)}$

\section{Ronco na população pediátrica}

A Academia Norte-Americana de Pediatria recomenda que crianças que roncam devam ser avaliadas, uma vez que a prevalência de ronco é superior a 10\% nessa população. 0 melhor teste para avaliar essas crianças roncadoras é através da polissonografia; porém, nessa população, há muita variação nos critérios utilizados para considerar um exame como normal ou anormal. ${ }^{(17)}$ Além disso, critérios clínicos isoladamente são insuficientes e incapazes de distinguir crianças com ronco primário ou SAHOS. Em crianças, de forma similar ao que acontece em adultos, acredita-se que o ronco primário seja uma fase inicial de um processo contínuo cujo outro extremo é caracterizado pela presença de SAHOS. ${ }^{(17-19)}$

Em um estudo, 2.209 crianças (com idades entre 9 e 15 anos) foram analisadas através de um estudo transversal. Foi encontrada uma prevalência de 5,6\% de crianças roncadoras, e os principais preditores de ronco nessa população pediátrica foram o aumento do peso corporal, a diminuição da patência nasal (rinite alérgica, desvio de septo e obstrução nasal) e condições clínicas que possam estreitar a via aérea superior (hipertrofia tonsilar, adenoidectomia sem tonsilectomia). A concentração de hemoglobina esteve aumentada em crianças roncadoras, sugerindo que essas crianças possam experimentar dessaturação da oxi-hemoglobina durante o sono. Além disso, o reconhecimento e o tratamento desses fatores de risco estão associados a uma melhor qualidade do sono, podendo, assim, prevenir o aparecimento de SAHOS nessas crianças. ${ }^{(18)}$

Nas crianças, a associação do ronco primário com complicações cardiovasculares também tem sido estudada. Em um estudo, 30 crianças com ronco primário tiveram pressão arterial sistêmica diurna aumentada e redução da distensibilidade arterial (avaliada pela velocidade de onda de pulso) em relação aos controles $(n=30) .{ }^{(19)}$

\section{Ronco nas gestantes}

A incidência de ronco (dados dos EUA) em mulheres grávidas saudáveis é de 14\%, 
significativamente maior do que em mulheres não grávidas. ${ }^{(20)}$ Durante a gravidez, surgem alterações na via aérea superior que podem explicar a maior prevalência de ronco e de outros distúrbios respiratórios do sono. Surgem reduções do diâmetro faríngeo, o que pode ser verificado clinicamente pelo escore de Mallampati. A patência nasal também está reduzida durante a gravidez, sendo que $42 \%$ das mulheres, por volta da $36^{a}$ semana de gestação, relatam congestão nasal e rinite alérgica. Esses aspectos podem estar relacionados ao aumento dos níveis de progesterona e de estrogênio conforme a gestação progride. A exata prevalência de ronco não é conhecida de forma precisa; porém, como na gestação há um aumento da incidência de ronco, possivelmente também há um aumento na incidência de SAHOS. Estudos têm indicado que SAHOS possa se desenvolver durante a gravidez em mulheres com tendência pré-existente e que a gravidade de SAHOS possa piorar em mulheres que, previamente à gravidez, já sofriam da doença. ${ }^{(20)}$

0 ronco durante a gravidez pode ser um importante achado e pode estar relacionado à presença de hipertensão arterial sistêmica materna e pré-eclampsia. Um dos mais importantes achados da pré-eclampsia éa reversão diurna do ritmo de pressão arterial (similar a SAHOS). ${ }^{(20)}$ Em sua maior parte, as mulheres grávidas com pré-eclampsia não apresentam, quando submetidas à polissonografia noturna, os achados clássicos de SAHOS, com episódios repetitivos de cessação completa ou parcial do fluxo aéreo e hipoxemia associada. Seus achados polissonográficos são mais compatíveis com SRVAS, mas a aplicação noturna de CPAP pode reduzir esses longos períodos noturnos de pressão arterial aumentada. ${ }^{(20)} \mathrm{A}$ polissonografia em mulheres grávidas pode ser realizada de forma similar àquela realizada em mulheres não grávidas. Contudo, dada a preferência de mulheres grávidas de dormir na posição lateral, é possível que o $1 \mathrm{AH}$ medido pela polissonografia seja subestimado. Além disso, também não existe evidência de que a polissonografia seja mais difícil de ser realizada em mulheres grávidas do que em não grávidas. (20)

Quanto às complicações fetais dos distúrbios respiratórios do sono durante a gravidez, os episódios repetitivos de eventos respiratórios obstrutivos durante o sono podem ocasionar episódios cíclicos de hipoxemia materna. A hipertensão arterial sistêmica e a vasoconstrição periférica estão comumente associadas a SAHOS, e essas complicações estão associadas a uma redução de oferta de oxigênio ao feto pela placenta. Assim, o ronco durante a gravidez pode levar a complicações fetais, como redução do crescimento intrauterino e baixo escore de Apgar ao nascimento. ${ }^{(20,21)}$ Outras complicações dos distúrbios respiratórios do sono na gravidez são a hipertensão arterial pulmonar e o diabetes mellitus gestacional. ${ }^{(20)} 0$ tratamento com CPAP tem sido utilizado em mulheres grávidas propiciando um bom prognóstico, reduzindo, inclusive, os incrementos da pressão arterial durante o sono em grávidas com pré-eclampsia. ${ }^{(20)}$ Em mulheres grávidas que desenvolvem pré-eclampsia, a circunferência do pescoço e a obesidade materna foram fatores de risco, na análise univariada, para hipertensão arterial sistêmica relacionada à gravidez e pré-eclampsia. A circunferência do pescoço $>34,7 \mathrm{~cm}$, contudo, foi um fator de risco independente tanto para a hipertensão arterial sistêmica relacionada à gravidez $(\mathrm{OR}=2,85$; 1C95\%: $1,51-5,37)$ e a pré-eclampsia $(O R=3,57$; 1C95\%: 1,43-8,88)..(21)

\section{Referências}

1. Gislason T, Aberg H, Taube A. Snoring and systemic hypertension--an epidemiological study. Acta Med Scand. 1987;222(5):415-21.

2. Koskenvuo M, Kaprio J, Partinen M, Langinvainio H, Sarna S, Heikkilä K. Snoring as a risk factor for hypertension and angina pectoris. Lancet. 1985;1(8434):893-6.

3. Koskenvuo M, Kaprio J, Telakivi T, Partinen M, Heikkilä K, Sarna S. Snoring as a risk factor for ischaemic heart disease and stroke in men. Br Med J (Clin Res Ed). 1987;294(6563):16-9.

4. Norton PG, Dunn EV. Snoring as a risk factor for disease: an epidemiological survey. Br Med J (Clin Res Ed). 1985;291(6496):630-2

5. Waller PC, Bhopal RS. Is snoring a cause of vascular disease? An epidemiological review. Lancet. 1989;1(8630):143-6.

6. Shepard JW Jr. Hypertension, cardiac arrhythmias, myocardial infarction, and stroke in relation to obstructive sleep apnea. Clin Chest Med. 1992;13(3):437-58.

7. Gainer JL. Hypoxia and atherosclerosis: re-evaluation of an old hypothesis. Atherosclerosis. 1987;68(3):263-6.

8. Hoffstein V. Is snoring dangerous to your health? Sleep. 1996;19(6):506-16.

9. Young T, Finn L, Hla KM, Morgan B, Palta M. Snoring as part of a dose-response relationship between sleep-disordered breathing and blood pressure. Sleep. 1996;19(10 Suppl):S202-5.

10. Young T, Palta M, Dempsey J, Skatrud J, Weber $\mathrm{S}$, Badr S. The occurrence of sleep-disordered 
breathing among middle-aged adults. N Engl J Med. 1993;328(17):1230-5.

11. Hu FB, Willett WC, Manson JE, Colditz GA, Rimm EB, Speizer FE, et al. Snoring and risk of cardiovascular disease in women. J Am Coll Cardiol. 2000;35(2):30813.

12. Zamarrón C, Gude F, Otero Otero Y, Rodríguez-Suárez JR. Snoring and myocardial infarction: a 4-year follow-up study. Respir Med. 1999;93(2):108-12.

13. Stradling JR, Crosby JH. Relation between systemic hypertension and sleep hypoxaemia or snoring: analysis in 748 men drawn from general practice. BMJ. 1990;300(6717):75-8.

14. Partinen M, Palomäki H. Snoring and cerebral infarction. Lancet. 1985;2(8468):1325-6.

15. Palomäki H, Partinen M, Juvela S, Kaste M. Snoring as a risk factor for sleep-related brain infarction. Stroke. 1989;20(10):1311-5.

16. Palomäki H. Snoring and the risk of ischemic brain infarction. Stroke. 1991;22(8):1021-5.
17. Section on Pediatric Pulmonology, Subcommittee on Obstructive Sleep Apnea Syndrome. American Academy of Pediatrics. Clinical practice guideline: diagnosis and management of childhood obstructive sleep apnea syndrome. Pediatrics. 2002;109(4):704-12.

18. Corbo GM, Forastiere F, Agabiti N, Pistelli R, Dell'Orco V, Perucci CA, et al. Snoring in 9- to 15-year-old children: risk factors and clinical relevance. Pediatrics. 2001;108(5):1149-54.

19. Kwok KL, Ng DK, Cheung YF. BP and arterial distensibility in children with primary snoring. Chest. 2003;123(5):1561-6.

20. Edwards N, Middleton PG, Blyton DM, Sullivan CE. Sleep disordered breathing and pregnancy. Thorax. 2002;57(6):555-8.

21. Ursavas A, Karadag M, Nalci N, Ercan 1, Gozu RO. Self-reported snoring, maternal obesity and neck circumference as risk factors for pregnancyinduced hypertension and preeclampsia. Respiration. 2008;76(1):33-9.

\section{Sobre os autores}

\section{Flávio José Magalhães da Silveira}

Diretor Médico. SLEEP - Laboratório de Estudo dos Distúrbios do Sono, Centro Médico BarraShopping, Rio de Janeiro (RJ) Brasil.

\section{Ricardo Luiz de Menezes Duarte}

Professor Convidado da Cadeira Cardiopulmonar. Universidade Federal do Estado do Rio de Janeiro - UNIRIO - e Diretor Médico. SLEEP - Laboratório de Estudo dos Distúrbios do Sono, Centro Médico BarraShopping, Rio de Janeiro (RJ) Brasil.

E-mail para contato: flaviomagalhaes1@gmail.com (F. Silveira) 